\title{
Toward new targets for the diagnosis and treatment of malignant diseases
}

\author{
Thematic issue dedicated to Theresia Thalhammer
}

Isabella Ellinger · Peter Pietschmann

Published online: 27 November 2014

(C) Springer-Verlag Wien 2014

Cancer remains among the leading causes of death worldwide; in the USA, the lifetime risk of developing (invasive) cancer for a woman is $38 \%$ and for a man is $43 \%$ [1]. Similar data are published for European countries. Alarmingly, the lifetime risk for persons in the UK to be diagnosed with cancer is projected to be $47 \%$ in 2030 [2]. Globally, the World Health Organization estimates that annual cancer cases will rise from 14 million in 2012 to 22 million within the next 2 decades [3]. In light of these numbers and the fact that malignant diseases impact dramatically on the patients, their families, and societies, it is a major aim of physicians and scientists to reduce the burden of cancer. In part, this already can be achieved at the individual level by avoiding or reducing major risk factors such as tobacco smoking or excessive sun exposure, unhealthy diets, or obesity, which account for more than $30 \%$ of cancer deaths. From the remaining cases, many would have a high chance to be cured if the tumor is detected early and treated adequately. However, we still lack tools for early diagnosis and treatment of malignant diseases, and this remains a research area with a huge potential of development.

We would like to dedicate this special issue of Wiener Medizinische Wochenschrift (WMW) to Associate Professor Theresia Thalhammer $\left(\mathrm{Mag}^{\mathrm{a}}, \mathrm{PhD}\right)$ on the occasion of her retirement. Theresia Thalhammer has devoted her professional life to find new targets for the diagnosis and treatment of malignant diseases. Striving for this goal, she has established many fruitful collaborations result-

Assoc. Prof. P. Pietschmann, MD $(\varangle)$ - Assoc. Prof. I. Ellinger, PhD Department of Pathophysiology and Allergy Research, Center for Pathophysiology, Infectiology and Immunology, Medical University of Vienna,

Währinger Gürtel 18-20,

1090 Vienna, Austria

e-mail: peter.pietschmann@meduniwien.ac.at ing in more than 100 peer-reviewed articles on this topic. This issue, composed of articles from six partners, summarizes current aspects of pathophysiology, diagnosis, and treatment of several types of cancers, illustrates the impact of research on clinical medicine and pinpoints directions of future research.

Theresia Thalhammer has studied pharmacy at the University of Vienna, but she has never been comfortable with the pure knowledge of currently available therapies. Her intrinsic spirit of research made her seek for new therapeutic possibilities. Upon receiving her final degree (Magistra), she started to work as a research assistant at the Department of Pathophysiology and Allergy Research (at that time, the Department of General and Experimental Pathology) at the Medical University of Vienna. She became a PhD student of Professor Jürg Graf (MD), a passionate explorer of the hepatobiliary system [4]. Theresia was introduced to the fascinating model system of the isolated perfused rat liver and started to investigate ion exchange processes and membrane transporters in hepatocytes. Rapidly, however, she was attracted by the idea to study aspects of cancer treatment in this versatile organ system.

During her stay as a Max Kade postdoctoral fellow of Robert E. Handschuhmacher at the Department of Pharmacy at Yale University in New Haven (USA) from 1990 to 1991, she characterized new members of the cyclophilin family in the rat liver. Members of this enzyme family are of interest for clinically relevant drug design, as they are targets of the drug cyclosporin, which is used as an immunosuppressant to treat patients following organ transplantation.

Upon her return to Vienna, Professor Maria Daoudaki (PhD; School of Medicine, Aristotle University of Thessaloniki, Greece) visited Theresia's laboratory to characterize hepatocytes isolated from normal and regenerated liver treated with different immunosuppressive drugs by 
FACS analysis. This turned out to be a fruitful collaboration, with funded projects from the Greek Ministry of Research to Maria Daoudaki and an academic collaboration (LLP-ERASMUS-Programm) between the Medical School of Aristotle University of Thessaloniki and the Medical University of Vienna, which continues until now. Maria Daoudaki and Ioannis Fouzas contribute a review to this issue that summarizes not only the intricate development of hepatocellular carcinoma but also its complexity with respect to research and therapy.

Soon thereafter, Theresia got into contact with Associate Professor Gerhard Hamilton (PhD; head of the Surgical Research Laboratories, Medical University of Vienna). Their first common publication in 1993 reported on the role of the MDR-1-encoded multiple drug resistance phenotype in prostate cancer cell lines. Now, more than 20 years later, Gerhard Hamilton and Barbara Rath discuss the limitations of chemotherapeutic interventions caused by the appearance of chemoresistance. While MDR-1 was a prime mover of chemoresistance, their article gives an overview on the many more molecules and pathways that are meanwhile known to be implicated in tumor resistance.

In 1995, Theresia received her „venia docendi,“ and became Associate Professor and head of the research group Cellular Pathology and Pharmacokinetics. Around that time, metabolism of anticancer drugs, specifically in the liver, became a common passion of Theresia and Walter Jäger. Associate Professor Walter Jäger (PhD; currently head of the Department of Clinical Pharmacy and Diagnostics, University of Vienna) and Theresia had already met in 1992. In 1997, they published their first common article on Amidox, an inhibitor of an enzyme required for de novo synthesis of DNA and therefore a target for cancer chemotherapy. In the following review article, Walter Jäger and coworkers detail the coordinated action of uptake transporters, metabolizing enzymes, and efflux pumps and recall their importance for alterations in drug disposal, adverse drug reactions, and drug-drug interactions.

In 1998, Theresia learnt to know Associate Professor Cem Ekmekcioglu (MD; Institute of Environmental Health, Medical University of Vienna), and they became interested in melatonin, the hormone of the darkness. This molecule with well-described functions in the circadian system also seems to participate as a modulating substance in many more cellular processes. Cem Ekmekcioglu and Theresia started their collaboration with evaluation of melatonin receptor expression in diverse organs, but soon became interested in the relationship of melatonin receptors and cancer. For this issue, Cem Ekmekcioglu summarized the current knowledge on the putative functions of melatonin receptors in malignant cells and tissues.

In 2006, Associate Professor Robert Zeillinger (PhD; Department of Obstetrics and Gynaecology, Medical University of Vienna) initiated the European Union Sixth Framework Program project OVCAD (Ovarian Cancer-
Diagnosis of a Silent Killer), which aimed at investigating new predictors for early detection of minimal residual disease in epithelial ovarian cancer. Among his Austrian project partners was Theresia. In this special issue of $W M W$, Stefanie Aust and Dietmar Pils, members of Robert Zeillinger's team and collaborators of Theresia, provide an overview on epithelial ovarian cancer biology.

One of the most recent collaborators of Theresia in the matter of cancer control is Professor Klaus Geissler (MD; head of the Fifth Department of Internal MedicineOncology/Hematology, Vienna and Ludwig Boltzmann Institute for Clinical Oncology, Hospital Hietzing). In 2011, Theresia and Klaus Geissler started a collaboration on embryonic signaling pathways in malignant diseases. In his article, Klaus Geissler discusses how results from basic science can find their way to practical applications in the clinic. His concise review on prominent examples for translational hematology summarizes perfectly what researchers such as Theresia aim for: the discovery of new mechanisms and molecules that-sooner or laterwill help to battle against the burden of cancer.

In addition to her Austrian and international collaborations, Theresia was very active in the mentoring of young scientists; during her career, she supervised more than 60 diploma and doctoral theses, and many of her students remained associated with research. She will be proud to see that she passed her enthusiasm and spirit of research to the next generation of researchers. Finally, we would like to thank Theresia Thalhammer for her contributions, her achievements, her dedication, and an excellent collaboration over many years. We wish her all the best for the years to come.

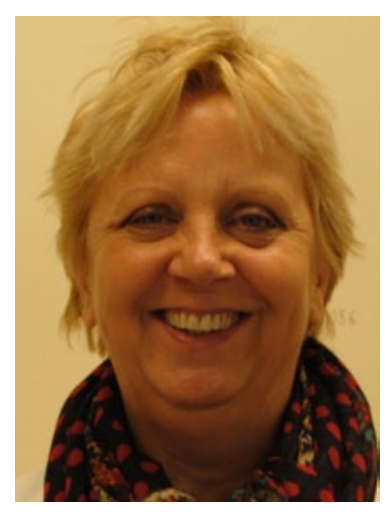

Associate Professor Theresia Thalhammer (Maga $\left.{ }^{\mathrm{a}} \mathrm{PhD}\right)$

\section{Conflict of interest}

The authors declare no conflict of interest.

\section{References}

1. Lifetime risk of developing or dying from cancer. 1.10.2014. http://www.cancer.org/cancer/cancerbasics/ lifetime-probability-of-developing-or-dying-from-cancer. Accessed 4 Nov. 2014. 
2. Lifetime risk of cancer. 26.7.2012. http://www.cancerresearchuk.org/cancer-info/cancerstats/incidence/risk/statistics-on-the-risk-of-developing-cancer. Accessed 4 Nov. 2014.

3. Cancer, Fact sheet No. 297. February 2014. http://www. who.int/mediacentre/factsheets/fs297/en/. Accessed 4 Nov. 2014
4. Fuchs R, Thalhammer T. Editorial: cell biology of the hepatobiliary system. Wien Med Wochenschr. 2008;158(19-20):531-3. 